\title{
A study of probable benefit of a bioresorbable polymer scaffold for safety and neurological recovery in patients with complete thoracic spinal cord injury: 6-month results from the INSPIRE study
}

\author{
Kee D. Kim, MD, ${ }^{1}$ K. Stuart Lee, MD, ${ }^{2}$ Domagoj Coric, MD, ${ }^{3}$ Jason J. Chang, MD, ${ }^{4}$ \\ James S. Harrop, MD, MSHQS, ${ }^{5}$ Nicholas Theodore, MD, ${ }^{6}$ and Richard M. Toselli, MD \\ 'Department of Neurological Surgery, UC Davis, Sacramento, California; '2Division of Neurosurgery, Vidant Health, Greenville, \\ North Carolina; ${ }^{3}$ Atrium Musculoskeletal Institute, Carolina NeuroSurgery \& Spine Associates, Charlotte, North Carolina; \\ ${ }^{4}$ Department of Neurological Surgery, Oregon Health and Sciences University, Portland, Oregon; ${ }^{5}$ Department of Neurological \\ and Orthopedic Surgery, Division of Spine and Peripheral Nerve Surgery, and Delaware Valley SCI Center, Thomas Jefferson \\ University, Philadelphia, Pennsylvania; ${ }^{6}$ Department of Neurosurgery, Johns Hopkins University, Baltimore, Maryland; and \\ ${ }^{7}$ InVivo Therapeutics Corp., Cambridge, Massachusetts
}

OBJECTIVE The aim of this study was to evaluate whether the investigational Neuro-Spinal Scaffold (NSS), a highly porous bioresorbable polymer device, demonstrates probable benefit for safety and neurological recovery in patients with complete (AIS grade A) T2-12 spinal cord injury (SCl) when implanted $\leq 96$ hours postinjury.

METHODS This was a prospective, open-label, multicenter, single-arm study in patients with a visible contusion on MRI. The NSS was implanted into the epicenter of the postirrigation intramedullary spinal cord contusion cavity with the intention of providing structural support to the injured spinal cord parenchyma. The primary efficacy endpoint was the proportion of patients who had an improvement of $\geq 1$ AIS grade (i.e., conversion from complete paraplegia to incomplete paraplegia) at the 6-month follow-up visit. A preset objective performance criterion established for the study was defined as an AIS grade conversion rate of $\geq 25 \%$. Secondary endpoints included change in neurological level of injury (NLI). This analysis reports on data through 6-month follow-up assessments.

RESULTS Nineteen patients underwent NSS implantation. There were 3 early withdrawals due to death, which were all determined by investigators to be unrelated to the NSS or the implantation procedure. Seven of 16 patients $(43.8 \%)$ who completed the 6 -month follow-up visit had conversion of neurological status (AIS grade A to grade $B$ [ $n=5]$ or $C$ [ $n=2]$ ). Five patients showed improvement in NLI of 1 to 2 levels compared with preimplantation assessment, 3 patients showed no change, and 8 patients showed deterioration of 1 to 4 levels. There were no unanticipated or serious adverse device effects or serious adverse events related to the NSS or the implantation procedure as determined by investigators.

CONCLUSIONS In this first-in-human study, implantation of the NSS within the spinal cord appeared to be safe in the setting of surgical decompression and stabilization for complete (AIS grade A) thoracic SCI. It was associated with a 6 -month AIS grade conversion rate that exceeded historical controls. The INSPIRE study data demonstrate that the potential benefits of the NSS outweigh the risks in this patient population and support further clinical investigation in a randomized controlled trial.

Clinical trial registration no.: NCT02138110 (clinicaltrials.gov)

https://thejns.org/doi/abs/10.3171/2020.8.SPINE191507

KEYWORDS spinal cord injury; AIS A; ASIA Impairment Scale grade A; Neuro-Spinal Scaffold implant; biopolymer; thoracic

ABBREVIATIONS ADE = adverse device effect; AIS = ASIA Impairment Scale; ASIA = American Spinal Injury Association; EMSCI = European Multicenter Study about Spinal Cord Injury; INSPIRE = InVivo Study of Probable Benefit of the Neuro-Spinal Scaffold for Safety and Neurological Recovery in Patients with Complete Thoracic Spinal Cord Injury; ISNCSCI = International Standards for Neurological Classification of SCI; NACTN = North American Clinical Trials Network; NLI = neurological level of injury; NSS = Neuro-Spinal Scaffold; SCI = spinal cord injury; SCIMS = Spinal Cord Injury Model Systems; TLICS = Thoracolumbar Injury Classification and Severity Score. SUBMITTED January 7, 2020. ACCEPTED August 17, 2020.

INCLUDE WHEN CITING Published online February 5, 2021; DOI: 10.3171/2020.8.SPINE191507. 
$\mathrm{M}$ ORE than 17,000 new cases of acute traumatic spinal cord injury (SCI) present to US hospitals each year, with an estimated 291,000 people currently living with chronic SCI..$^{1-4} \mathrm{SCI}$ can be devastating to the individual involved but may also have an adverse effect on the family with a huge societal cost. Estimated lifetime costs can vary, but they may amount to millions of dollars. ${ }^{2}$

The primary SCI is most often caused by direct mechanical trauma resulting in spinal cord contusion or compression. ${ }^{5}$ The secondary injury cascade exacerbates damage within the spinal cord parenchyma. Substantial necrosis immediately follows the primary mechanism and the effects are compounded by continuous neuronal and oligodendroglial apoptosis. ${ }^{5,6}$ Throughout the secondary injury, substantial loss of myelin has also been noted. ${ }^{5,7}$ These secondary injury mechanisms exacerbate the severity of the injury. Treatment standards for SCI have experienced little improvement over recent years, and current treatment options fail to effectively repair or mitigate these destructive processes. ${ }^{5,8}$

Based on the International Standards for Neurological Classification of SCI (ISNCSCI), an SCI classified as American Spinal Injury Association (ASIA) Impairment Scale (AIS) grade A is considered to be complete (i.e., no motor or sensory function is preserved in the sacral segments [S4-5]). ${ }^{9}$ This is the most severe grade of SCI, with complete loss of motor and sensory function below the level of injury. Only a small percentage of patients who sustain a complete SCI ever regain full neurological function. ${ }^{10}$

The Neuro-Spinal Scaffold (NSS; InVivo Therapeutics Corp.) is an investigational device that has been developed as an adjunct to standard of care for the treatment of SCI. The NSS is a highly porous bioresorbable polymer composed of poly(lactic-co-glycolic acid)-b-poly-(L-lysine) (Fig. 1), ${ }^{11}$ a material widely used in US FDA-approved devices, and is designed to provide structural support to injured spinal cord and a supportive matrix for endogenous healing and repair processes following implantation within a spinal cord contusion. In a rat contusion model, NSS implantation reduced cyst volume, spared white matter, and promoted generation of remodeled tissue at 12 weeks versus controls. ${ }^{12}$ Remodeled tissue was rich in neuropermissive extracellular matrix and facilitated endogenous sprouting.

This paper presents the 6-month results from the InVivo Study of Probable Benefit of the Neuro-Spinal Scaffold for Safety and Neurological Recovery in Patients with Complete Thoracic Spinal Cord Injury (INSPIRE).

\section{Methods}

\section{Study Design and Oversight}

INSPIRE was a prospective, open-label, single-arm, multicenter study. The NSS has been designated as a Humanitarian Use Device by the US FDA, which requires evidence that probable benefit to health from use of the device outweighs the risk of injury or illness from its use. The first case from this study demonstrating feasibility of implanting the NSS directly into the injured spinal cord of a human was reported previously.$^{13}$ An interim evaluation performed in the first 5 patients who underwent NSS implantation and completed 6-month postimplantation assessment supported conversion of the study into a pivotal Humanitarian Device Exemption probable benefit study.

The study expansion had a planned total enrollment of up to 30 patients (including patients assessed in the interim evaluation) to achieve 20 patients in the primary endpoint analysis set. The study was put on hold in July 2017 in discussion with the US FDA following 3 deaths that occurred within 2 weeks of implantation; all were determined by investigators to be unrelated to the NSS or its implantation procedure. Enrollment was closed, with 16 patients meeting the primary endpoint analysis criteria.

Key enrollment criteria implemented at the study sites included the following: age $\geq 16$ years to $\leq 70$ years, traumatic nonpenetrating SCI (contusion injury) no less than 4 $\mathrm{mm}$ in diameter on MRI, AIS grade A classification, neurological level of injury (NLI) at T2-12, and requirement for open spine surgery $\leq 96$ hours of SCI (as part of standard treatment) allowing access to the dura overlying the injured spinal cord for NSS implantation. Key exclusion criteria were complete transection of the spinal cord or more than one discrete SCI on MRI, significant traumatic brain injury or coma, terminal illness, and clinically significant preexisting neurological or respiratory comorbidities. Full patient eligibility criteria are provided in Table 1.

This study was conducted in accordance with the ethical principles of the Declaration of Helsinki, International Conference on Harmonization guidelines for Good Clinical Practice, and applicable regulatory requirements. All patients provided signed written informed consent prior to enrollment in the study or undergoing any study procedure. The protocol and all relevant study forms were approved by the respective institutional review board/research ethics board of each institution. An independent Data and Safety Monitoring Board provided recommendations. This study was registered at ClinicalTrials.gov (identifier NCT02138110).

\section{Screening Assessments, Study Procedures, and NSS Implantation}

Neurological status was assessed by the investigator or a designated trained medical professional using the ISNCSCI ${ }^{9}$ and ASIA 2015 worksheet at the following time points: screening; within 8 hours prior to open spine surgery (to confirm a reliable ISNCSCI examination and AIS grade A classification); at 24, 48, and 72 hours postimplantation; at hospital discharge; and at 1, 2, 3, 6, 12, and 24 months postimplantation. MRI studies without contrast were performed at screening and at 72 hours and 3, 6, 12, and 24 months postimplantation. Long-term follow-up including general annual health assessment and safety event monitoring continues annually via phone for 3 to 10 years postimplantation.

The ISNCSCI examinations determined sensory and motor levels (left and right sides), NLI, sensory and motor scores, and AIS grade. ${ }^{9}$ The injury was considered complete (AIS grade A) when there was no motor or sensory function in the lowest sacral segments (S4-5). Incomplete injuries were defined as follows: AIS grade B (sensory 

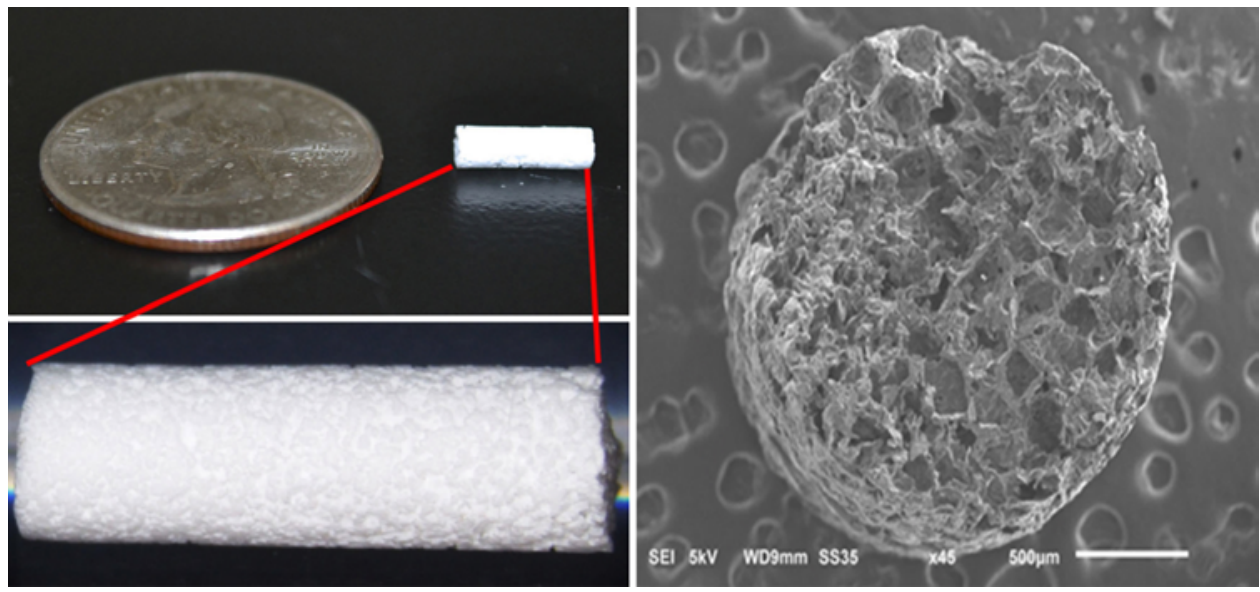

FIG. 1. The porous, cylindrical NSS comes in 2 sizes, 2-mm diameter $\times 10-\mathrm{mm}$ length and 3-mm diameter $\times 10$-mm length. The NSS is designed for optimal fit in the intraspinal postirrigation cavity and can be trimmed, if necessary, to a specific length. The NSS is expected to be resorbed from the site of implantation within 4-8 weeks. Left: Macroscopic view of a representative $3 \times$ $10-\mathrm{mm}$ NSS. Right: Scanning electron microscopy cross-sectional image of a representative $2 \times 10-\mathrm{mm}$ NSS. Bar $=500 \mu \mathrm{m}$. Reprinted from Layer RT, Ulich TR, Coric D, et al. New clinical-pathological classification of intraspinal injury following traumatic acute complete thoracic spinal cord injury: postdurotomy/myelotomy observations from the INSPIRE trial. Neurosurgery. 2017;64(CN_suppl_1):105-109, by permission of Oxford University Press. Copyright @ 2017 by the Congress of Neurological Surgeons. All rights reserved. For permissions, please email journals.permissions@oup.com.

incomplete-sensory but not motor function is preserved below the level of injury and includes sacral segments S4-5), AIS grade C (motor incomplete-motor function preserved below level of injury; voluntary anal contraction or sparing of motor function 3 levels below injury), and AIS grade D (motor incomplete-as for grade C, but with at least half of key muscles below injury functioning against gravity). AIS grade $\mathrm{E}$ indicated normal function. NLI refers to the most caudal segment of the spinal cord with normal sensory and motor function on both sides of the body. Sensory scores were assessed on a scale from 0 to 2 for each sensory point tested on each side of the body ( pinprick maximum score $=112$ and light-touch maximum score $=112$ ), and motor scores were assessed on a scale from 0 to 5 for each myotome tested on each side of the body (upper-limb maximum score $=50$ and lowerlimb maximum score $=50$ ), with higher scores indicating better neurological function.

Open spine surgery was performed (bony decompression, reduction, and/or stabilization). On visualization of the dura, intraoperative ultrasonography was performed to localize the area of maximal damage as initially assessed by preoperative MRI. Durotomy and myelotomy, if needed, were performed to expose the intramedullary contusion site. The contusion cavity was then irrigated with isotonic saline solution to wash away any superficial hemorrhagic material and devitalized tissue. The NSS was soaked in isotonic saline solution and, if necessary, trimmed at one end to the size needed to fit the postirrigation contusion cavity without causing any undue tension on the surrounding spinal cord. The NSS was then gently implanted lengthwise into the epicenter of the intraspinal contusion cavity followed by dural closure. Based on preclinical testing, the NSS is expected to be essentially resorbed (mass loss of $\geq 85 \%$ ) from the site of implant within 4 to 8 weeks (InVivo Therapeutics Corp., unpub- lished data). All patients were treated according to standard of care at a qualified trauma center and participated in a comprehensive rehabilitation program following hospital discharge.

Representative examples of an MR image demonstrating the preoperative hematoma (Fig. 2), preimplantation and postimplantation ultrasound images (Fig. 3), and an intraoperative video demonstrating myelotomy and implantation of the NSS (Video 1) are provided.

VIDEO 1. Case 14. Intraoperative video of myelotomy and implantation of the NSS in a patient with complete $\mathrm{SCl}$ at T2. Copyright InVivo Therapeutics Corp. Published with permission. Click here to view.

\section{Outcomes}

The primary efficacy endpoint was the proportion of patients who had an improvement from preimplantation of $\geq 1$ AIS grade at the 6-month follow-up visit. An objective performance criterion established for the study was defined as achievement of conversion from complete paraplegia (AIS grade A) to incomplete paraplegia by 6 months in $\geq 25 \%$ of patients. This preset benchmark was derived from the natural history rate in a similar patient population from the European Multicenter Study about Spinal Cord Injury (EMSCI) database (15.6\%). ${ }^{14}$

Secondary endpoints included changes in NLI, sensory scores, and motor scores (all measured using the ISNCSCI classification tool), and changes in spinal cord anatomy as determined by MRI.

The Medical Dictionary for Regulatory Activities (MedDRA) Version 17.0 (March 2014) was used to classify all safety events.

\section{Statistical Analysis}

The primary endpoint analysis set includes all patients 


\begin{tabular}{|c|c|}
\hline Inclusion Criteria & Exclusion Criteria \\
\hline $\begin{array}{l}\text { 1) AIS grade A classification of traumatic } \\
\text { SCl at T2-12 neurological level of } \\
\text { injury confirmed by a qualified medical } \\
\text { professional } \\
\text { 2) Recent injury (must receive the NSS } \\
\text { within } 96 \text { hrs from injury) } \\
\text { 3) Nonpenetrating SCI (contusion injury) } \\
\text { that is no less than } 4 \text { mm in diameter } \\
\text { by MRI } \\
\text { 4) Requires open spine surgery allowing } \\
\text { access to the injured spinal cord (pa- } \\
\text { tients requiring either posterior surgical } \\
\text { approach or posterior plus anterior } \\
\text { approach will be eligible) } \\
\text { 5) Informed consent obtained } \\
\text { 6) } 16-70 \text { yrs of age, inclusive } \\
\text { 7) Hemodynamically stable and deemed a } \\
\text { suitable candidate for surgery }\end{array}$ & 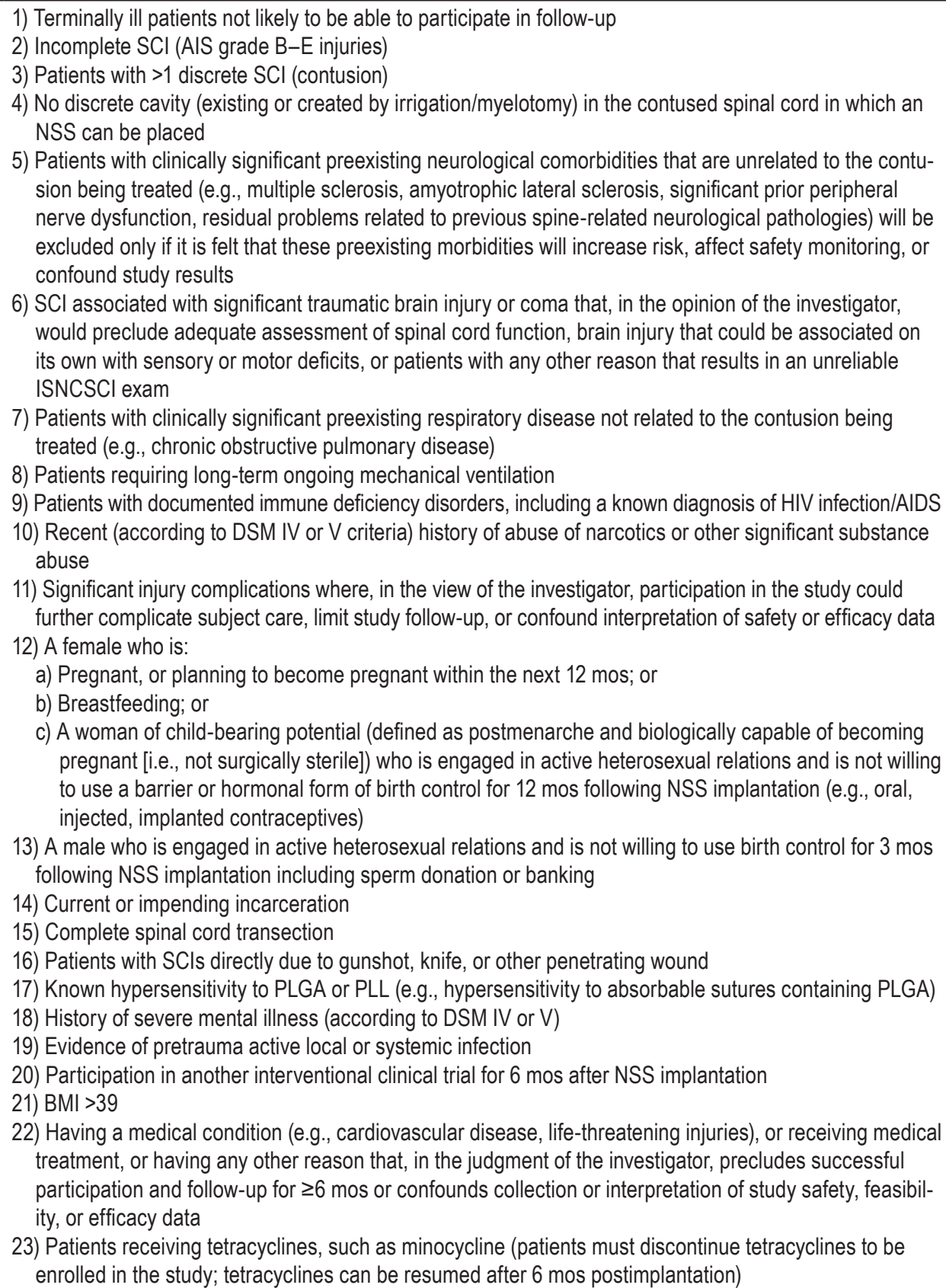 \\
\hline
\end{tabular}

DSM = Diagnostic and Statistical Manual of Mental Disorders; PLGA = poly(lactic-co-glycolic acid); PLL = poly-L-lysine.

who underwent NSS implantation with no major protocol deviations and had completed the 6-month primary endpoint follow-up visit. A sensitivity analysis imputed a value for any missing AIS grade at the 6-month followup visit as not having an improvement of $\geq 1$ AIS grade. Efficacy and safety analyses are reported for all treated patients.

The preimplantation ISNCSCI examination performed within 8 hours before surgery was used as the baseline value for ISNCSCI measurements, and the screening MRI was used as the baseline value for changes in spinal cord anatomy.

These results represent a preplanned analysis including data through the 6-month follow-up visit (data cutoff January 10, 2019). Data were analyzed according to the latest protocol version approved by the US FDA prior to database lock using SAS for Windows (version 9.3, SAS Institute Inc.). 


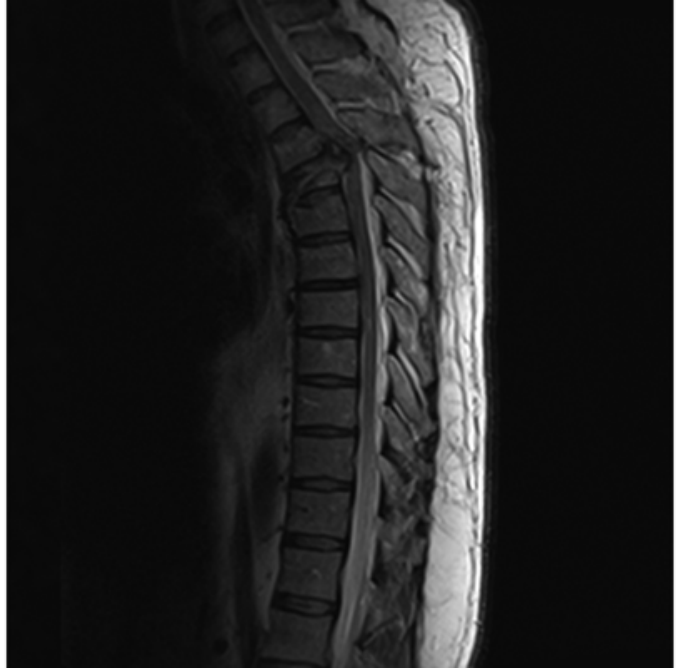

FIG. 2. Case 9. Preoperative sagittal T2-weighted MR image showing an upper-thoracic burst fracture at $\mathrm{T} 3$ with complete $\mathrm{SCI}$

\section{Results}

Between October 13, 2014, and June 23, 2017, 25 patients were enrolled at 12 study sites in North America (11 sites in the US and 1 in Canada). Nineteen patients underwent NSS implantation, 5 patients did not meet eligibility criteria, and 1 patient was enrolled but not treated with the NSS, and durotomy was not performed. There were 3 early withdrawals due to death determined by investigators to be unrelated to the NSS or its implantation.

\section{Patient Demographics and Baseline Characteristics}

Patient demographic and baseline characteristics for enrolled patients are presented in Table 2. Among patients who underwent NSS implantation $(\mathrm{n}=19), 8$ had an upperthoracic injury (T2-5), 6 had a mid-thoracic injury (T6-9), and 5 had a lower-thoracic injury (T10-12). All patients had AO Spine type B2 or C fracture, ${ }^{15}$ and all required surgical intervention as assessed according to the Thoracolumbar Injury Classification and Severity Score (TLICS) ${ }^{16}$ classification system. The mean time from injury to start of surgery was 40.9 hours (range 7-81 hours); most patients underwent surgery within 72 hours postinjury $(n=16)$, including 6 patients within 24 hours and 2 within 8 hours.

\section{Primary Efficacy Analysis}

Seven of the 16 patients (43.8\%) who completed the 6 -month follow-up visit converted to AIS grade B $(n=5)$ or $\mathrm{C}(\mathrm{n}=2)$.

AIS grades at each time point for patients who were implanted with the NSS are provided in Table 3. Among patients who achieved the 6-month primary endpoint, 3 had initial conversion by the 1 -month visit (AIS grade $\mathrm{B}, \mathrm{n}=1$; AIS grade $C, n=2), 2$ patients had converted to AIS grade $\mathrm{B}$ by the 2-month visit, and 1 patient each had converted to AIS grade B by the 3- and 6-month visits, respectively. Two patients experienced improvement and deterioration in AIS grade over time: 1 patient converted to AIS grade $\mathrm{B}$ by the 2-month visit due to the presence of sacral sensation, reverted back to AIS grade $A$ at the 3-month visit, and then converted again to AIS grade B by the 6-month visit due to the presence of deep anal pressure. Another patient converted to AIS grade C by the 1-month visit due to the presence of voluntary anal contraction and deep anal pressure, and reverted to AIS grade B at the 3-month visit due to the loss of voluntary anal contractions, and then converted back to AIS grade $\mathrm{C}$ at the 6-month visit due to sacral sensation and to the presence of both deep anal pressure and voluntary anal contraction. An additional patient had conversion to AIS grade B at the 1-month visit but was assessed as AIS grade A at future assessments.

A sensitivity analysis including all NSS-implanted patients $(n=19)$ resulted in a 6-month AIS grade conversion rate of $36.8 \%$.
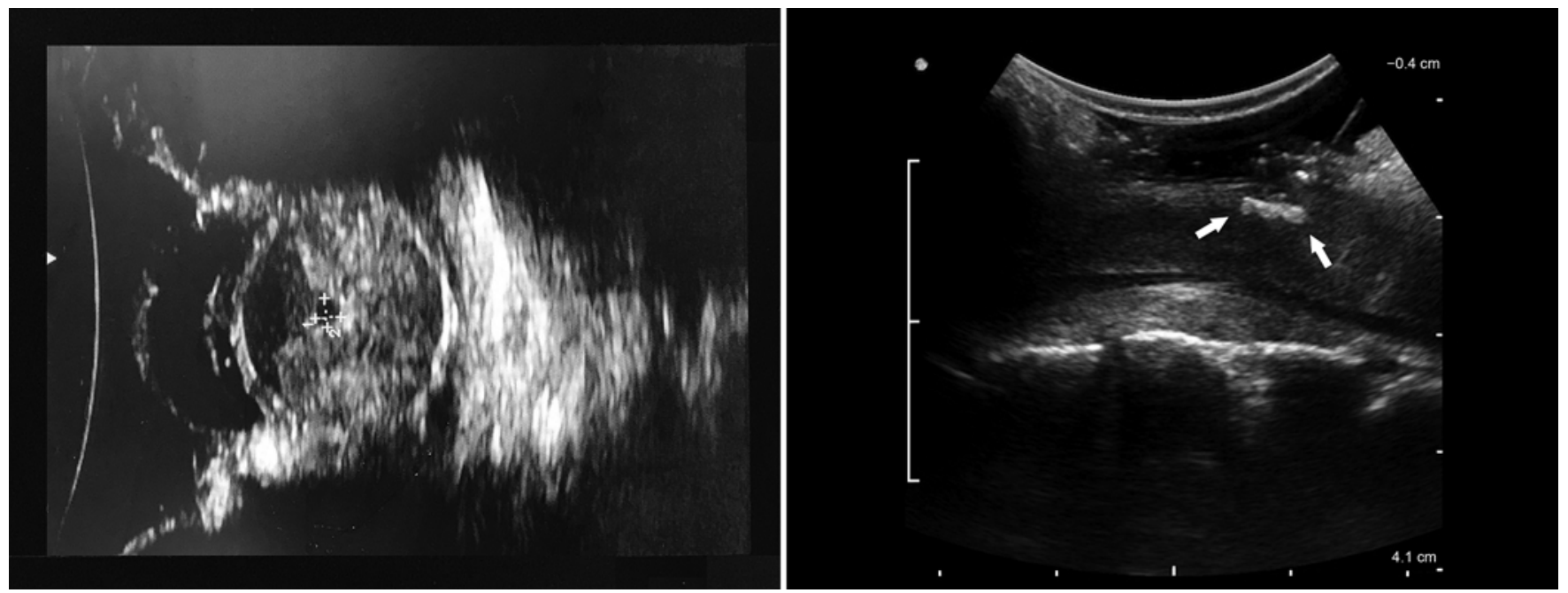

FIG. 3. Left: Case 18. Preoperative ultrasound study obtained in a patient with complete SCI at T12. Right: Case 5. Intraoperative postimplantation ultrasound study obtained in a patient with complete SCl at T4. The arrows indicate the implanted intramedullary NSS. 
TABLE 2. Baseline demographics and cause of injury for all enrolled patients who were included after screening

\begin{tabular}{lc}
\hline \multicolumn{1}{c}{ Characteristic } & NSS $(\mathrm{n}=20)$ \\
\hline Mean age (range), years & $36(18-69)$ \\
\hline Sex, $\mathrm{n}(\%)$ & $4(20.0)$ \\
Female & $16(80.0)$ \\
Male & \\
\hline Race, $\mathrm{n}(\%)$ & $1(5.0)$ \\
American Indian or Alaskan Native & $2(10.0)$ \\
Black or African American & $16(80.0)$ \\
White & $1(5.0)$ \\
Other & \\
\hline Ethnicity, $\mathrm{n}$ (\%) & $4(20.0)$ \\
Hispanic or Latino & $16(80.0)$ \\
Non-Hispanic or Latino & $172(147-185)$ \\
\hline Mean height (range), cm & $83(58-127)$ \\
\hline Mean weight (range), kg & $28(20-38)$ \\
\hline Mean BMl (range), kg/m² & \\
\hline Cause of injury, $\mathrm{n}(\%)$ & $5(25.0)$ \\
Fall & $1(5.0)$ \\
Other: all-terrain vehicle & $14(70.0)$ \\
Vehicular &
\end{tabular}

\section{Secondary Efficacy Analyses}

Changes in NLI are presented in Table 4. At the 6-month follow-up visit, 5 patients showed an improvement in NLI of 1 to 2 levels compared with preimplantation, 3 patients showed no change, and 8 patients showed a deterioration of 1 to 4 levels.

Among patients who completed the 6-month follow-up visit $(n=16)$, the mean (SD) change in sensory pinprick score was an improvement of 0.9 (5.5) points compared with preimplantation. Ten patients demonstrated an improvement ranging from 1 to 10 points, and 6 patients demonstrated a deterioration ranging from -2 to -12 points. Sensory pinprick score changes varied over time; 7 patients had improved sensory pinprick scores over time, 4 patients had decreased scores over time, and 5 patients experienced fluctuating scores.

Changes in sensory light-touch scores were generally consistent with sensory pinprick scores. The mean (SD) change in sensory light-touch score among patients who completed the 6-month follow-up visit was an improvement of 2.9 (5.7) points compared with preimplantation. Ten patients demonstrated an improvement in sensory light-touch score compared with preimplantation (1 to 13 points), 4 patients demonstrated a deterioration ( -2 to -8 points), and 2 patients demonstrated no change. Sensory light-touch scores were consistent with pinprick scores, except for 1 patient who had an improvement in sensory lighttouch score and a deterioration in sensory pinprick score at the 2-month follow-up visit, and 1 patient who had a deterioration in sensory light-touch score and an improvement in sensory pinprick score at the 6-month follow-up visit.

Fourteen patients had no change in motor scores at the 6-month follow-up visit compared with preimplantation, indicating no change in motor function and no lower-ex- tremity motor function present. One patient demonstrated an improvement in total motor score of 10 points at the 6-month follow-up visit. An additional patient experienced improvement in motor scores in the lower extremities, but the total change was not reportable secondary to the lowerextremity fractures at screening and preimplantation preventing proper testing according to the ISNCSCI. However, a motor score of 61 at the 6-month follow-up visit indicated a minimal improvement of 10 points over the preimplantation motor score of 51 .

MRI showed the presence of cysts in 5 of 14 patients with evaluable images at the 6-month follow-up visit, which were first observed at the 3 -month visit in 3 patients. One patient had documented spinal cord adhesion at the 6-month visit, and another patient had a documented syrinx on MRI at the 3-month visit with a decrease in size noted at the 6-month visit. None of these events required any additional surgical intervention.

\section{Safety}

The following safety events not related to the NSS or its implantation procedure occurred in more than $20 \%$ of patients who underwent NSS implantation $(\mathrm{n}=19)$ : urinary tract infection $(68.4 \%)$, muscle spasms $(57.9 \%)$, neuralgia (47.4\%), pyrexia (42.1\%), abdominal distention (31.6\%), leukocytosis (31.6\%), back pain (26.3\%), pain in extremity $(26.3 \%)$, pleural effusion (26.3\%), arthralgia (21.1\%), atelectasis $(21.1 \%)$, constipation $(21.1 \%)$, decubitus ulcer $(21.1 \%)$, depression $(21.1 \%)$, hypophosphatemia (21.1\%), hypotension $(21.1 \%)$, incision site pain $(21.1 \%)$, muscle spasticity (21.1\%), and urinary incontinence (21.1\%).

There were 22 serious adverse events; none were deemed by investigators to be related to the NSS or its implantation procedure. Intensity was mild for 1 event, moderate for 10 events, severe for 10 events, and not provided for 1 event (decrease in neurological function). The most common serious event was urinary tract infection $(n=3)$, followed by 2 events each of pleural effusion, pulmonary embolism, respiratory distress, and respiratory failure. One serious event was reported for each of the following: cellulitis, Clostridium difficile colitis, postoperative wound infection, sepsis, traumatic pneumothorax, dehydration, necrotizing myositis, CSF leakage, cerebrovascular accident, spinal cord disorder, and deep vein thrombosis. Three deaths determined by investigators not to be related to the NSS or procedure to implant the NSS were reported (1 each due to sepsis, cerebrovascular accident, and pulmonary embolism). There were no other adverse events leading to discontinuation from the study.

No serious or unanticipated adverse device effects (ADEs) were reported. Two ADEs determined to be possibly related to the NSS and the implantation procedure occurred in 1 patient (mild intermittent nerve pain and a single event of mild myelomalacia). An additional 4 ADEs considered to be related to the implantation procedure were reported (severe intermittent increase in generalized pain, moderate continuous declining neurological status, mild continuous acute postoperative pulmonary insufficiency, and moderate continuous incisional pain). There were 2 adverse events of interest in a single patient (thoracic CSF leak of moderate intensity and deep wound infection of 
TABLE 3. AIS grades in patients who underwent NSS implantation

\begin{tabular}{|c|c|c|c|c|c|c|c|c|c|}
\hline \multirow[b]{2}{*}{$\begin{array}{l}\text { Case } \\
\text { No. }\end{array}$} & \multirow[b]{2}{*}{$\begin{array}{l}\text { Age } \\
\text { Group }\end{array}$} & \multirow[b]{2}{*}{$\begin{array}{l}\text { Preimplantation } \\
\qquad \mathrm{NLI}(\mathrm{n}=19)\end{array}$} & \multirow{2}{*}{$\begin{array}{l}\text { Time From } \\
\text { Injury to } \\
\text { Implantation } \\
\text { (hrs) }\end{array}$} & \multicolumn{6}{|c|}{ AIS Grade } \\
\hline & & & & $\begin{array}{l}\text { Preimplantation } \\
\qquad(\mathrm{n}=19)\end{array}$ & $\begin{array}{l}\text { Hospital } \\
\text { Discharge } \\
(n=16)\end{array}$ & $\begin{array}{c}1 \mathrm{Mo} \\
\text { Postimplantation } \\
(n=15)\end{array}$ & $\begin{array}{l}2 \text { Mos } \\
\text { Postimplantation } \\
\quad(n=12)\end{array}$ & $\begin{array}{c}3 \text { Mos } \\
\text { Postimplantation } \\
\quad(n=16)\end{array}$ & $\begin{array}{c}6 \text { Mos } \\
\text { Postimplantation } \\
\quad(n=16)\end{array}$ \\
\hline $1^{*}$ & Adult & T3† & 70 & $A$ & & & & & \\
\hline 2 & Adult & $\mathrm{T} 12$ & 67 & A & A & A & B & $A$ & B \\
\hline 3 & Adult & $\mathrm{T} 11$ & 9 & A & A & C & Not donef & C & C \\
\hline 4 & Adult & $\mathrm{T} 7$ & 46 & A & A & A & Not donef & A & A \\
\hline 5 & Adult & T4 & 83 & A & A & B & B & B & B \\
\hline 6 & Adult & T3 & 22 & A & A & A & A & A & A \\
\hline $7^{*}$ & Adult & T4 & 21 & A & & & & & \\
\hline 8 & Pediatric§ & $\mathrm{T} 10$ & 21 & A & A & B & Not done & A & A \\
\hline 9 & Adult & T3 & 47 & A & A & A & A & A & A \\
\hline 10 & Pediatric§ & $\mathrm{T} 10$ & 9 & A & Not done & $A$ & B & B & $B$ \\
\hline 11 & Adult & T8 & 37 & A & A & A & A & $A$ & A \\
\hline 12 & Adult & T9 & 77 & A & A & A & A & A & A \\
\hline 13 & Pediatric§ & T8 & 69 & A & $\mathrm{A} \rrbracket$ & A & A & A & B \\
\hline 14 & Adult & $\mathrm{T} 2$ & 79 & A & A & Not done & Not done & A & A \\
\hline 15 & Adult & T9 & 29 & A & $\mathrm{A} \rrbracket$ & A & A & A & A \\
\hline 16 & Adult & T3 & 53 & A & A & A & A & A & A \\
\hline 17 & Adult & T4 & 40 & A & A & A & A & B & B \\
\hline $18^{*}$ & Adult & $\mathrm{T} 12$ & 28 & A & A & & & & \\
\hline 19 & Adult & T6 & 20 & A & A & $C$ & C & $B$ & $C$ \\
\hline
\end{tabular}

* Died prior to hospital discharge; no further follow-up visits.

$\dagger \mathrm{T} 3$ at screening assessment; preimplantation assessment not done.

$\ddagger$ Two-month visit not required per protocol approved at the time.

$\S$ Defined as $\leq 21$ years old.

ๆ Hospital discharge visit occurred after 1-month visit.

moderate intensity), both of which resolved. Two patients had a deterioration in NLI of $>2$ dermatome levels compared with the preimplantation visit on 2 successive examinations $\geq 8$ hours apart. However, the Data and Safety Monitoring Board did not recommend stopping the study for either of these cases after careful and thorough review.

\section{Discussion}

\section{Key Findings}

The INSPIRE study describes the safety and feasibility of NSS implantation in patients with acute complete (AIS grade A) thoracic SCI and reports a 6-month AIS grade conversion rate that exceeded the study objective performance criterion $(\geq 25 \%)$. Seven of 16 evaluable patients had AIS conversion from complete paraplegia to incomplete injuries classified as either AIS grade B (5 patients) or C (2 patients).

\section{Comparison With Natural History}

SCI is a devastating injury, and there are few treatment options for patients beyond standard of care, which include decompression, stabilization, and fusion. ${ }^{17,18}$ The NSS has shown a potential benefit when compared with the natural history of these injuries. The AIS grade conversion rate in the current study (44\%) was greater than historical bench- marks (approximately 16\%) for patients with T2-12 complete (AIS grade A) SCI from large natural history databases (EMSCI and the Spinal Cord Injury Model Systems [SCIMS]). ${ }^{14,19}$ A recent study (CONTEMPO) evaluating outcomes for standard-of-care comparator groups from the North American Clinical Trials Network (NACTN), EMSCI, and SCIMS registries, with inclusion and exclusion criteria that closely matched those of INSPIRE, included 170 patients who sustained injuries between 2006 and 2016. ${ }^{20}$ The spontaneous conversion rate at approximately 6 months postinjury or beyond was $21.1 \%$ (weighted average for all registries combined), which is consistent with earlier benchmarks and provides further support to the relevance of the current study results.

In the INSPIRE study, conversion to incomplete paraplegia was reported in a higher proportion of patients with lower-thoracic injuries (T10-12; 3 of 4 patients [75.0\%]) than those with upper-thoracic (T2-5; 2 of 6 patients [33.3\%]) or mid-thoracic (T6-9; 2 of 6 patients [33.3\%]) injuries, which is in line with what can be expected based on natural history data. ${ }^{14,19,20}$ However, it should be noted that the patient distribution in the INSPIRE study did not favor lower-level injuries, as only approximately $25 \%$ of patients who underwent NSS implantation had T10-12 injuries. The estimated lifetime hospitalization costs for thoracic AIS grade B patients are substantially less than 
TABLE 4. NLI in patients who underwent NSS implantation

\begin{tabular}{|c|c|c|c|c|c|c|c|c|}
\hline \multirow[b]{2}{*}{$\begin{array}{l}\text { Case } \\
\text { No. }\end{array}$} & \multirow[b]{2}{*}{ Age Group } & \multirow{2}{*}{$\begin{array}{l}\text { Time From } \\
\text { Injury to } \\
\text { Implantation } \\
\text { (hrs) }\end{array}$} & \multicolumn{6}{|c|}{$\mathrm{NLI}$} \\
\hline & & & $\begin{array}{l}\text { Preimplantation } \\
\qquad(n=19)\end{array}$ & $\begin{array}{l}\text { Hospital } \\
\text { Discharge } \\
(n=17)\end{array}$ & $\begin{array}{c}1 \mathrm{Mo} \\
\text { Postimplantation } \\
(n=15)\end{array}$ & $\begin{array}{c}2 \text { Mos } \\
\text { Postimplantation } \\
\quad(n=12)\end{array}$ & $\begin{array}{c}3 \text { Mos } \\
\text { Postimplantation } \\
(n=16)\end{array}$ & $\begin{array}{c}6 \text { Mos } \\
\text { Postimplantation } \\
(n=16)\end{array}$ \\
\hline $1^{*}$ & Adult & 70 & T3† & & & & & \\
\hline 2 & Adult & 67 & $\mathrm{~T} 12$ & L1 & L1 & L1 & L1 & L1 \\
\hline 3 & Adult & 9 & T11 & T11 & L1 & Not donef & L1 & L1 \\
\hline 4 & Adult & 46 & $\mathrm{~T} 7$ & T5 & T5 & Not donef & T5 & T5 \\
\hline 5 & Adult & 83 & T4 & $\mathrm{T} 4$ & T4 & T3 & T5 & T4 \\
\hline 6 & Adult & 22 & T3 & T3 & T3 & T3 & $\mathrm{T} 2$ & T2 \\
\hline $7^{*}$ & Adult & 21 & T4 & & & & & \\
\hline 8 & Pediatric§ & 21 & $\mathrm{~T} 10$ & $\mathrm{~T} 7$ & T8 & Not done & $\mathrm{T7}$ & T6 \\
\hline 9 & Adult & 47 & T3 & T3 & T3 & T3 & T3 & $\mathrm{T} 4$ \\
\hline 10 & Pediatric§ & 9 & $\mathrm{~T} 10$ & T9 & $\mathrm{T} 10$ & $\mathrm{~T} 10$ & T9 & T9 \\
\hline 11 & Adult & 37 & T8 & T9 & T10 & T10 & T10 & T10 \\
\hline 12 & Adult & 77 & T9 & T10 & T10 & T10 & T10 & T10 \\
\hline 13 & Pediatric§ & 69 & T8 & T8ף & $\mathrm{T} 7$ & $\mathrm{~T} 7$ & $\mathrm{~T} 7$ & $\mathrm{~T} 7$ \\
\hline 14 & Adult & 79 & T2 & $\mathrm{T} 2$ & Not done & Not done & T2 & $\mathrm{C} 8$ \\
\hline 15 & Adult & 29 & T9 & T9ף & T9 & T9 & T9 & T9 \\
\hline 16 & Adult & 53 & T3 & T3 & T3 & T3 & T3 & T3 \\
\hline 17 & Adult & 40 & T4 & $\mathrm{T} 4$ & $\mathrm{~T} 4$ & $\mathrm{~T} 4$ & $\mathrm{~T} 4$ & T3 \\
\hline $18^{*}$ & Adult & 28 & $\mathrm{~T} 12$ & $\mathrm{~T} 12$ & & & & \\
\hline 19 & Adult & 20 & T6 & T3 & T5 & $\mathrm{T} 4$ & $\mathrm{~T} 4$ & $\mathrm{~T} 4$ \\
\hline
\end{tabular}

* Died prior to hospital discharge; no further follow-up visits.

† T3 at screening assessment; preimplantation assessment not done.

$\ddagger$ Two-month visit not required per protocol approved at the time.

$\S$ Defined as $\leq 21$ years old.

ๆ Hospital discharge visit occurred after 1-month visit.

for AIS grade A patients, and the risk for SCI-related complications such as pressure ulcers is reduced..$^{21,22}$

\section{Benefits of the NSS Over Decompression and Myelotomy Alone}

Decompression surgery alone can provide benefit to patients, with a trend being seen for increased recovery with earlier surgery. ${ }^{23,24}$ The NSS is intended to serve as an extracellular matrix aiming to provide support to surrounding spared tissue after injury, minimize expansion of areas of necrosis, and support endogenous healing and repair processes following injury, and may harbor the promise of sparing of white matter, increasing neural sprouting, and decreasing posttraumatic cyst formation. ${ }^{12}$ In a rat contusion model, benefits of NSS implantation over myelotomy alone (allowing spontaneous extrusion and irrigation of necrotic debris and relieving spinal cord pressure) included new tissue formation, almost no cavitation, and an extensive region of SC-derived P0-positive myelin in the spared tissue, suggesting that implantation of the NSS may provide additional benefit beyond simple decompression and myelotomy. ${ }^{12}$

\section{Timing of Decompression}

Balancing the benefits of early surgery against the risks of operative intervention in critically ill patients is an important issue for physicians treating patients with SCI. Although there are no definitive guidelines regarding the optimal time to decompression, ${ }^{24,25}$ there has been a trend over the past 10-15 years to perform decompressive surgery earlier. The INSPIRE study reflects the current standard of care, which favors early spinal decompression and stabilization. All patients underwent surgery within 96 hours of injury. No formal statistical analysis was performed; however, there did not appear to be any correlation between time to surgery and AIS grade conversion.

\section{Contusion- and Compound-Type Injuries}

Early postdurotomy observations from the INSPIRE study provided visual evidence of 2 forms of cord damage: injuries where the cord surface is intact (contusion type) and injuries where the pia is breached and there is visible but incomplete cord parenchymal separation (compound type). ${ }^{11}$ Successful NSS implantation and subsequent AIS grade improvements were observed in patients with both injury types in this study.

\section{Secondary Endpoints}

The NLI results in the current study generally showed 
1- and 2-level variations that were not clinically significant. These may be explained by either fluctuation in sensory level seen in the natural history of SCI or the variability in testing related to the thoracic dermatomes.

The sensory changes in the study align with the natural history of recovery in this patient population. Although valuable in understanding the complete neurological status of the patient, it is difficult to conclude any positive or negative effects of changes in sensory scores.

Although motor function is believed to be a valuable endpoint due to its ability to lead to ambulation, its utility as an assessment tool following thoracic injuries may be limited. Spontaneous motor improvement following complete thoracic injuries is extremely limited, and the chance of observing a meaningful therapeutic effect is low. ${ }^{26}$ Lee et al. reported an average lower-extremity motor score change of 1.5 to 4.1 points, depending on initial NLI, with a median change of $0 .{ }^{19}$ Zariffa et al. published an average 2-point improvement in this patient population. ${ }^{14}$

Cysts, spinal cord adhesions, and the syrinx in NSStreated patients, as documented on MRI, are consistent with the natural history of the SCI population. These events did not require any additional surgical intervention.

\section{Safety of the NSS}

There were no unanticipated ADEs and no major morbidity related to the NSS or the procedure to implant the NSS as determined by the investigators in this study. All 3 safety events deemed possibly related to the device were of mild severity. There were 2 safety events of interest in a single patient (CSF leak and infection), which were likely related to the severity of the original injury that included a defect in the dura. Overall, the safety events reported in the INSPIRE study were consistent with what would be expected in the routine care of SCI patients. ${ }^{27}$

\section{Study Limitations and Future Directions}

This study had several limitations. The study was nonblinded and nonrandomized with no comparator group, and patient numbers were small (as can be expected given the inherent enrollment challenges associated with this patient population). ${ }^{28,29}$ Patients continue to be followed in this study, including ISNCSCI assessments through 24 months postinjury, and a separate randomized, controlled, single-blind, multicenter study has been initiated to evaluate whether the NSS is safe and demonstrates probable benefit in the treatment of complete (AIS grade A) thoracic SCI versus standard-of-care open spine surgery (INSPIRE 2.0; ClinicalTrials.gov identifier NCT03762655).

\section{Conclusions}

In this first-in-human study, implantation of the NSS within the spinal cord appeared to be safe in the setting of surgical decompression and stabilization for complete (AIS grade A) thoracic SCI. It was associated with a 6-month AIS grade conversion rate that exceeded historical controls. The INSPIRE study data demonstrate that the potential benefits of the NSS outweigh the risks in this patient population and support further clinical investigation in a randomized controlled trial.

\section{Acknowledgments}

We thank the additional study investigators who contributed to data collection: Kumar Kakarla, MD (Barrow Neurological Institute, Phoenix, Arizona), William Bockenek, MD (Carolina Neurosurgery \& Spine Associates, Charlotte, North Carolina), Patrick Hsieh, MD (Department of Neurological Surgery, University of Southern California, Los Angeles, California), and Steven S. Yocom, MD (Department of Neurosurgery, Cooper Neurological Institute, Camden, New Jersey); appreciate the continued support of Dr. Robert Langer for the INSPIRE trials and his commitment to improving the quality of life of SCI patients; and thank Nikki Moreland of Nous Healthcare Communications Ltd. for medical writing and editorial support funded by InVivo Therapeutics Corp.

\section{References}

1. Jain NB, Ayers GD, Peterson EN, et al. Traumatic spinal cord injury in the United States, 1993-2012. JAMA. 2015;313(22): 2236-2243.

2. National Spinal Cord Injury Statistical Center. Facts and figures at a glance, updated 2020. Accessed October 14, 2020. https://www.nscisc.uab.edu/

3. Saunders LL, Selassie A, Cao Y, et al. Epidemiology of pediatric traumatic spinal cord injury in a population-based cohort, 1998-2012. Top Spinal Cord Inj Rehabil. 2015;21(4): 325-332.

4. Selvarajah S, Hammond ER, Haider AH, et al. The burden of acute traumatic spinal cord injury among adults in the United States: an update. J Neurotrauma. 2014;31(3):228-238.

5. Ashammakhi N, Kim HJ, Ehsanipour A, et al. Regenerative therapy for spinal cord injury. Tissue Eng Part B Rev. 2019; 25(6):471-491.

6. Baptiste DC, Fehlings MG. Update on the treatment of spinal cord injury. Prog Brain Res. 2007;161:217-233.

7. Crowe MJ, Bresnahan JC, Shuman SL, et al. Apoptosis and delayed degeneration after spinal cord injury in rats and monkeys. Nat Med. 1997;3(1):73-76.

8. Courtine G, Sofroniew MV. Spinal cord repair: advances in biology and technology. Nat Med. 2019;25(6):898-908.

9. Kirshblum SC, Burns SP, Biering-Sorensen F, et al. International standards for neurological classification of spinal cord injury (Revised 2011). J Spinal Cord Med. 2011;34(6): 535-546.

10. Fawcett JW, Curt A, Steeves JD, et al. Guidelines for the conduct of clinical trials for spinal cord injury as developed by the ICCP panel: spontaneous recovery after spinal cord injury and statistical power needed for therapeutic clinical trials. Spinal Cord. 2007;45(3):190-205.

11. Layer RT, Ulich TR, Coric D, et al. New clinical-pathological classification of intraspinal injury following traumatic acute complete thoracic spinal cord injury: postdurotomy/myelotomy observations from the INSPIRE trial. Neurosurgery. 2017;64(CN_suppl_1):105-109.

12. Guest JD, Moore SW, Aimetti AA, et al. Internal decompression of the acutely contused spinal cord: Differential effects of irrigation only versus biodegradable scaffold implantation. Biomaterials. 2018;185:284-300.

13. Theodore N, Hlubek R, Danielson J, et al. First human implantation of a bioresorbable polymer scaffold for acute traumatic spinal cord injury: a clinical pilot study for safety and feasibility. Neurosurgery. 2016;79(2):E305-E312.

14. Zariffa J, Kramer JLK, Fawcett JW, et al. Characterization of neurological recovery following traumatic sensorimotor complete thoracic spinal cord injury. Spinal Cord. 2011;49(3): 463-471.

15. Reinhold M, Audigé L, Schnake KJ, et al. AO spine injury classification system: a revision proposal for the thoracic and lumbar spine. Eur Spine J. 2013;22(10):2184-2201. 
16. Vaccaro AR, Lehman RA Jr, Hurlbert RJ, et al. A new classification of thoracolumbar injuries: the importance of injury morphology, the integrity of the posterior ligamentous complex, and neurologic status. Spine (Phila Pa 1976). 2005; 30(20):2325-2333.

17. Anderson PA, Raksin PB, Arnold PM, et al. Congress of Neurological Surgeons systematic review and evidence-based guidelines on the evaluation and treatment of patients with thoracolumbar spine trauma: surgical approaches. Neurosurgery. 2019;84(1):E56-E58.

18. Martin AR, Aleksanderek I, Fehlings MG. Diagnosis and acute management of spinal cord injury: current best practices and emerging therapies. Curr Trauma Rep. 2015;1: 169-181.

19. Lee BA, Leiby BE, Marino RJ. Neurological and functional recovery after thoracic spinal cord injury. J Spinal Cord Med. 2016;39(1):67-76.

20. Aimetti AA, Kirshblum S, Curt A, et al. Natural history of neurological improvement following complete (AIS A) thoracic spinal cord injury across three registries to guide acute clinical trial design and interpretation. Spinal Cord. 2019; 57(9):753-762.

21. Dukes EM, Kirshblum S, Aimetti AA, et al. Relationship of American Spinal Injury Association Impairment Scale grade to post-injury hospitalization and costs in thoracic spinal cord injury. Neurosurgery. 2018;83(3):445-451.

22. Brienza D, Krishnan S, Karg P, et al. Predictors of pressure ulcer incidence following traumatic spinal cord injury: a secondary analysis of a prospective longitudinal study. Spinal Cord. 2018;56(1):28-34.

23. Fehlings MG, Vaccaro A, Wilson JR, et al. Early versus delayed decompression for traumatic cervical spinal cord injury: results of the Surgical Timing in Acute Spinal Cord Injury Study (STASCIS). PLoS One. 2012;7(2):e32037.

24. Fehlings MG, Tetreault LA, Wilson JR, et al. A clinical practice guideline for the management of acute spinal cord injury: introduction, rationale, and scope. Global Spine J. 2017;7(3) (suppl):84S-94S.

25. Eichholz KM, Rabb CH, Anderson PA, et al. Congress of Neurological Surgeons systematic review and evidence-based guidelines on the evaluation and treatment of patients with thoracolumbular spine trauma: timing of surgical intervention. Neurosurgery. 2019;84(1):E53-E55.

26. Steeves JD. Bench to bedside: challenges of clinical translation. Prog Brain Res. 2015;218:227-239.

27. Jiang F, Jaja BNR, Kurpad SN, et al. Acute adverse events after spinal cord injury and their relationship to long-term neurologic and functional outcomes: analysis from the North American Clinical Trials Network for spinal cord injury. Crit Care Med. 2019;47(11):e854-e862.

28. Blight AR, Hsieh J, Curt A, et al. The challenge of recruitment for neurotherapeutic clinical trials in spinal cord injury. Spinal Cord. 2019;57(5):348-359.

29. Lee RS, Noonan VK, Batke J, et al. Feasibility of patient recruitment into clinical trials of experimental treatments for acute spinal cord injury. J Clin Neurosci. 2012;19(10): $1338-1343$.

\section{Disclosures}

This study was sponsored by InVivo Therapeutics Corp.

Dr. Kim has served as a member of an advisory board (INSPIRE study steering committee) for InVivo Therapeutics Corp.; received research funding from Empiric Spine, Fziomed, InVivo Therapeu- tics Corp., Medtronic, Orthofix, Seikagaku, Vertex, and Zimmer Biomet Spine; been a consultant for Corentec, Globus, Vertex, and Zimmer Biomet; received royalties from Precision Spine and Zimmer Biomet Spine; and serves on the Board of Directors and holds stock options at Molecular Matrix. Dr. Lee has served as a member of an advisory board (INSPIRE study steering committee) for InVivo Therapeutics Corp. Dr. Coric has received research funding from InVivo Therapeutics Corp.; been a consultant for Medtronic, Premia Spine, Spinal Kinetics, Spine Wave, and Stryker; received royalties from RTI Surgical and Spine Wave; is a stockholder with Spinal Kinetics, Spine Wave, and Premia Spine; and has served as an advisory board member for United Health Care. Dr. Theodore has received research funding from the Department of Defense and InVivo Therapeutics Corp.; has been a consultant for Globus Medical; and directly owned and received royalties from DePuy Synthes and Globus Medical. Dr. Harrop has been a consultant for DePuy Synthes and Ethicon and served as an advisor for Abbvie and AlaMab Therapeutics. Dr. Toselli serves as Chief Medical Officer/Chief Executive Officer and holds stock options at InVivo Therapeutics Corp.

\section{Author Contributions}

Conception and design: Theodore. Acquisition of data: Kim, Lee, Coric, Chang, Harrop, Theodore. Analysis and interpretation of data: all authors. Drafting the article: Kim. Critically revising the article: all authors. Reviewed submitted version of manuscript: all authors. Approved the final version of the manuscript on behalf of all authors: Kim.

\section{Supplemental Information Videos}

Video 1. https://vimeo.com/463130852.

\section{Previous Presentations}

Portions of this work were presented in abstract/oral presentation form at the 2018 AANS Annual Scientific Meeting, New Orleans, Louisiana, April 30-May 2, 2018, and in abstract/e-poster presentation form at the 2019 AANS Annual Scientific Meeting, San Diego, California, April 13-17, 2019.

\section{Correspondence}

Kee D. Kim: UC Davis Health, Sacramento, CA. kdkim@ ucdavis.edu. 